$4^{\text {th }}$ International Meeting on Calcitonin Gene-Related Peptide (CGRP)

TheScientificWorld (2001) 1(S1), 20

ISSN 1532-2246; DOI 10.1100/tsw.2001.428

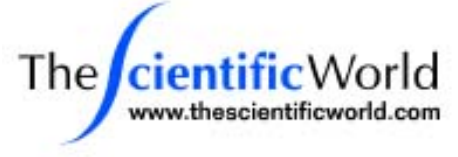

\title{
ROLE OF CGRP IN SENSITIZATION OF DURA MATER
}

\author{
K. Messlinger \\ Department of Physiology and Experimental Pathophysiology, University of Erlangen-Nuernberg, \\ 91054 Erlangen, Germany
}

The mammalian dura mater encephali is richly supplied by trigeminal nerve fibers, a considerable proportion of which contains calcitonin gene-related peptide (CGRP). As plasma levels of CGRP are increased in some forms of headaches, the question is in which way CGRP is involved in nociceptive mechanisms within the peripheral and the central trigeminovascular system.

CGRP is released in animal preparations upon electrical and chemonociceptive stimulation of trigeminal afferents in vivo and in vitro. In a hemisected rat skull preparation, CGRP release from the cranial dura mater was increased by inflammatory mediators, lowering of $\mathrm{pH}$, and nitric oxide. Following electrophysiological recording, CGRP immunoreactive nerve fibers were found in mechanosensitive receptive fields of dural afferents around meningeal arteries. Different lines of evidence suggest that CGRP has a key position in several vasodilatory mechanisms of the cranial dura mater. Laser Doppler flow measurements have shown that the vasodilatory effect of nitric oxide in the rat dura mater is partly mediated through CGRP. Prostaglandins and histamine released from dural mast cells may also be linked to the vasodilatory activity of CGRP.

Although CGRP release seems not to activate meningeal afferents directly, it may constitute an important principle for the sensitization of trigeminal nociceptors. If CGRP acts as a neuromodulator in the spinal trigeminal nucleus, the coupling of nitric oxide and CGRP may additionally provide an effective mechanism of central sensitization. 

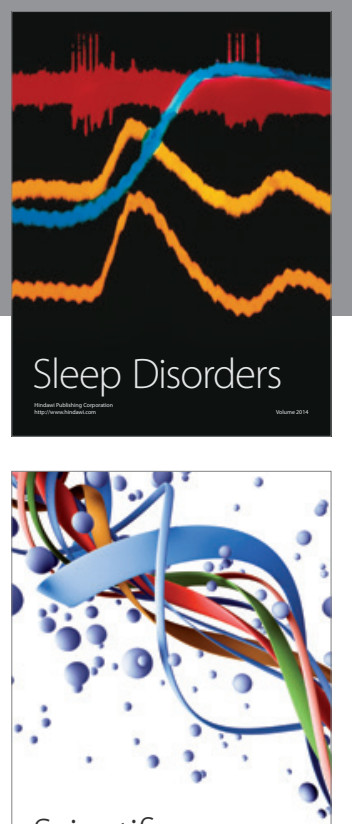

Scientifica
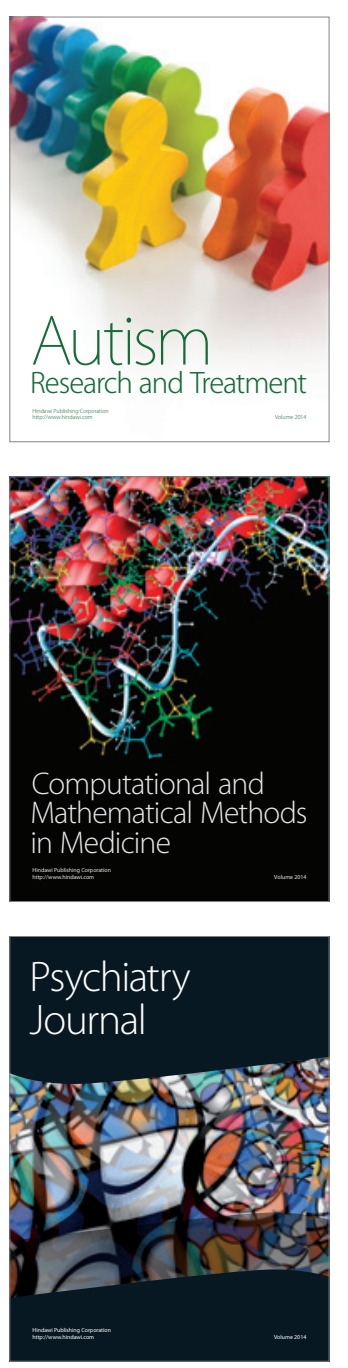
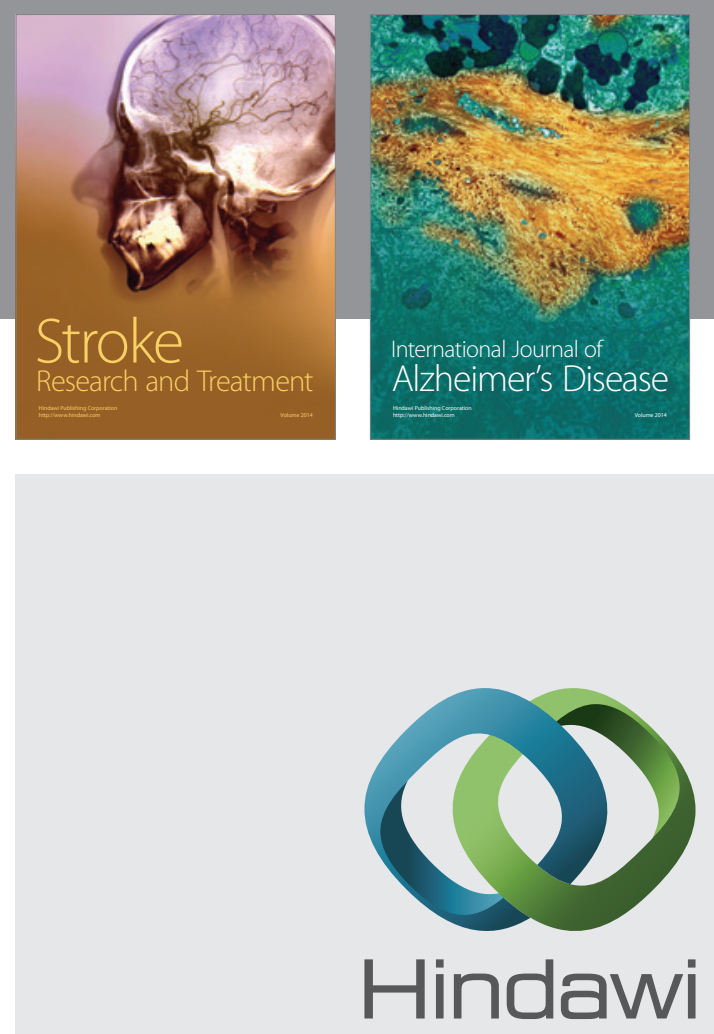

Submit your manuscripts at

http://www.hindawi.com
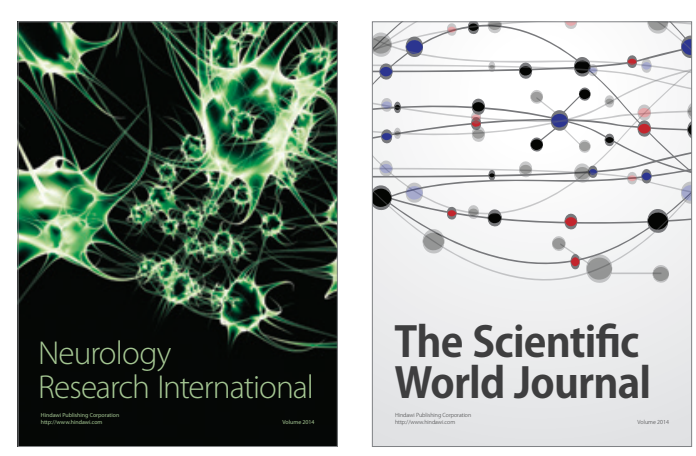

The Scientific World Journal

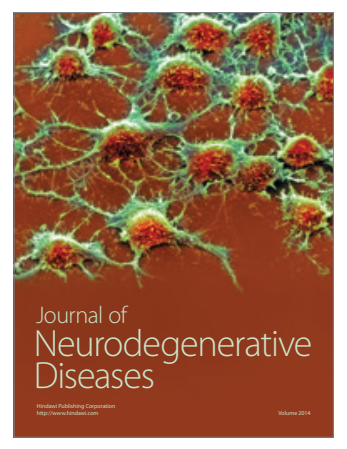

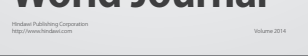

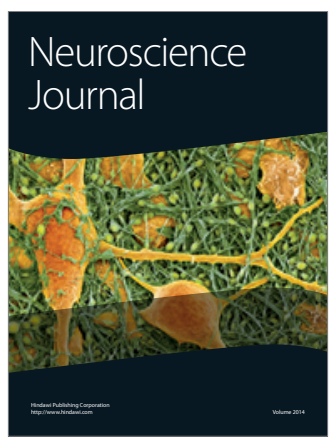

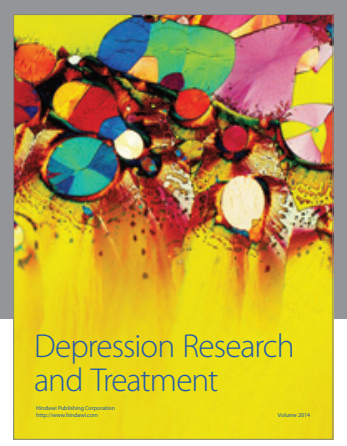
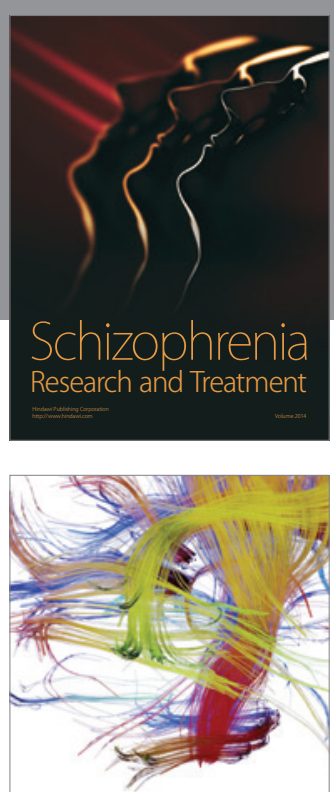

Brain Science

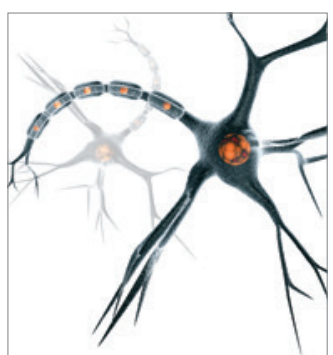

Neural Plasticity
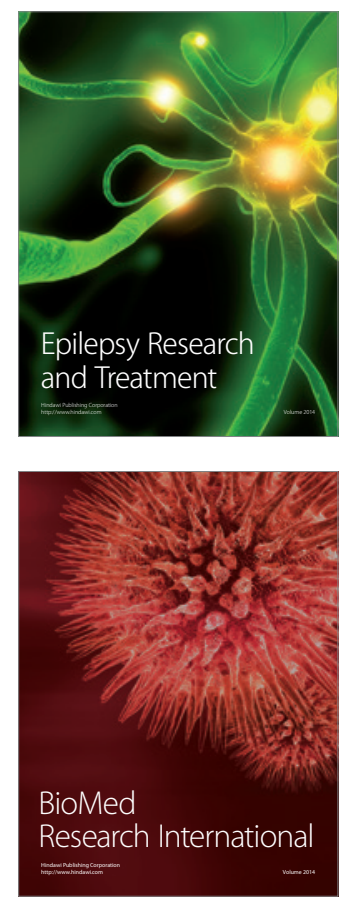

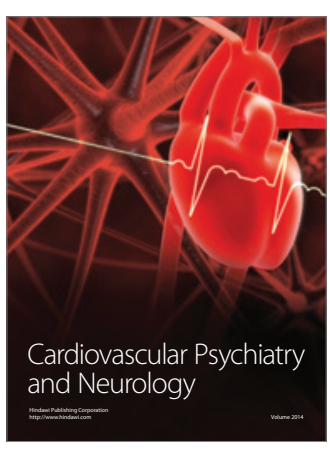

Parkinson's

Disease
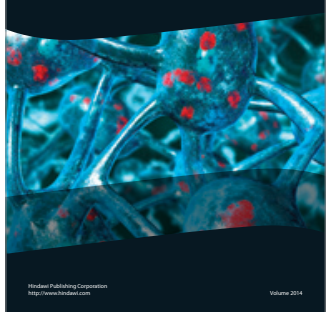